PASJ: Publ. Astron. Soc. Japan , 1-??,

(C) 2021. Astronomical Society of Japan.

\title{
Identifying Subclasses of Long Gamma-Ray Bursts with Cumulative Light Curve Morphology of Prompt Emissions
}

\author{
Ryo Tsutsui, ${ }^{1}$ Takashi Nakamura, ${ }^{2}$ Daisuke Yonetoku, ${ }^{3}$ Keitaro Takahashi, ${ }^{4}$ and Yoshiyuki Morihara ${ }^{3}$ \\ ${ }^{1}$ Research Center for the Early Universe, School of Science, University of Tokyo, Bunkyo-ku, Tokyo 113-0033, Japan \\ ${ }^{2}$ Department of Physics, Kyoto University, Kyoto 606-8502, Japan \\ ${ }^{3}$ Department of Physics, Kanazawa University, Kakuma, Kanazawa, Ishikawa 920-1192, Japan \\ ${ }^{4}$ Faculty of Science, Kumamoto University, Kurokami, Kumamoto, 860-8555, Japan
}

(Received ; accepted )

\begin{abstract}
We argue a new classification scheme of long gamma-ray bursts (LGRBs) using the morphology of the cumulative light curve of the prompt emission. We parametrize the morphology by the absolute deviation from their constant luminosity $(A D C L)$ and derive the value for 36 LGRBs which have spectropic redshifts, spectral parameters determined by the Band model, 1-second peak fluxes, fluences, and 64-msec resolution light curves whose peak counts are 10 times larger than background fluctuations. Then we devide the sample according to the value of ADCL into two groups $(A D C L<0.17$ and $A D C L>0.17)$ and, for each group, derive the spectral peak energy $E_{\mathrm{p}}$ - peak luminosity $L_{\mathrm{p}}$ correlation and the Fundamental Plane of LGRBs, which is a correlation between the spectral peak energy $E_{\mathrm{p}}$, the luminosity time $T_{\mathrm{L}}\left(\equiv E_{\text {iso }} / L_{\mathrm{p}}\right.$ where $E_{\text {iso }}$ is isotropic energy) and the peak luminosity $L_{\mathrm{p}}$. We find that both of the correlations for both groups are statistically more significant compared with ones derived from all samples. The Fundamental Planes with small and large ADCL are given by $L_{\mathrm{p}}=10^{52.53 \pm 0.01}\left(E_{\mathrm{p}} / 10^{2.71} \mathrm{keV}\right)^{1.84 \pm 0.03}\left(T_{\mathrm{L}} / 10^{0.86} \mathrm{sec}\right)^{0.29 \pm 0.08}$ with $\chi_{\nu}^{2}=10.93 / 14$ and $L_{\mathrm{p}}=10^{52.98 \pm 0.08}\left(E_{\mathrm{p}} / 10^{2.71} \mathrm{keV}\right)^{1.82 \pm 0.09}\left(T_{\mathrm{L}} / 10^{0.86} \mathrm{sec}\right)^{0.85 \pm 0.27}$ with $\chi_{\nu}^{2}=7.58 / 8$, respectively. This fact implies the existence of subclasses of LGRBs characterized by the value of $A D C L$. Also there is a hint for the existence of the intermediate- $A D C L$ class which deviates from both fundamental planes. Both relations are so tight that our result provides a new accurate distance measurement scheme up to the high redshift universe.
\end{abstract}

Key words: gamma rays: bursts — gamma rays: observations — gamma rays: cosmology

\section{Introduction}

In spite of the discovery of the observational diversity of the afterglow, parent galaxy, and environment properties of gamma-ray bursts (GRBs) in Swift era, the prompt emission studies over the last 10 years have not succeeded in revealing telltale GRB subclasses beyond the shorthard and long-soft classification (Kouveliotou et al. 1993). Except for some unusually low luminous events which are often associated with Type Ic Supernovae (e.g., 980425, 060218), all long gamma-ray bursts (LGRBs) seem to have similar properties and no subclasses.

In Tsutsui et al. (2009), we found a correlation between the spectral peak energy $E_{\mathrm{p}}$, the luminosity time $T_{\mathrm{L}}$ and the peak luminosity $L_{\mathrm{p}}$ (Fundamental Plane ${ }^{1}$ of LGRBs). However, the correlation has a relatively large number of outliers $(\sim 30 \%)$ and they deteriorate the usefulness of LGRBs as standard candles which probe the expansion history of universe (Tsutsui et al. 2011). The outliers may

\footnotetext{
The Fundamental Plane is a two-dimensional family first discovered between velocity dispersion, mean surface brightness and luminosity of elliptical galaxy (Djorgovski \& Davis 1987). The term is used for various two-dimensional families of other objects, such as galaxy clusters (Schaeffer et al. 1993), black hole activity (Merloni et al. 2003) and X-ray gas in normal galaxies (Diehl \& Statler 2005).
}

also imply the existence of subclasses and if we can identify them it would not only reduce the ambiguity of the correlation but also give some information on the origin of LGRBs.

In this paper, we show the statistical significance of the correlation improves by considering the morphology of the cumulative light curve of the prompt emission. Specifically, first, we define the absolute deviation from their constant luminosity ( $A D C L$ ) of the cumulative light curve. Then we derive the correlation both for small and large $A D C L$ groups and they are shown to be tighter than one derived by all LGRBs. We also find that dimmer outliers of Tsutsui et al. (2011) have intermediate $A D C L$ values.

\section{Data Description}

Since the discovery of a correlation between the peak energy $E_{\mathrm{p}}$ and the isotropic energy $E_{\text {iso }}$ (Amati et al. 2002), several correlations between $E_{\mathrm{p}}$ and other brightness (Yonetoku et al. 2004; Ghirlanda et al. 2004a), were proposed. There are also some studies to improve these correlations by adding third parameters of GRBs (Liang \& Zhang 2005; Firmani et al. 2006; Tsutsui et al. 2009; Yu et al. 2009). These correlations are thought to be important not only to investigate the nature of their emis- 
sion mechanism but also to measure distance up to the high redshift universe where no other distance indicators have been observed (Ghirlanda et al. 2004b; Schaefer 2007; Kodama et al. 2008; Liang et al. 2008; Cardone et al. 2009; Tsutsui et al. 2009).

In spite of their attraction, there have been many debate against these correlations (Nakar \& Piran 2005; Band \& Preece 2005; Butler et al. 2007; Rossi et al. 2008; Collazzi \& Schaefer 2008; Shahmoradi \& Nemiroff 2011).

Most of these discussions, however, are based on incomplete data without redshifts, and data with many systematic errors which conceal the nature of GRBs. Therefore, we do not take these works into account in this paper.

We have investigated origins of systematic errors of the spectral - brightness correlations (Yonetoku et al. 2010; Tsutsui et al. 2010), and succeeded to remove them (Tsutsui et al. 2011). As a result, we have found that the $E_{\mathrm{p}}-T_{\mathrm{L}}-L_{\mathrm{p}}$ correlation possibly divides LGRBs into at least three subclasses. In this paper, we study a new classification scheme based on a relationship between the residual from the $E_{\mathrm{p}}-T_{\mathrm{L}}-L_{\mathrm{p}}$ correlation and long term pulse shape within the whole duration of bursts.

Before we study the classification of LGRBs, here we update and recompile the database of Yonetoku et al. (2010) and Tsutsui et al. (2011). There are more than 120 GRBs with known redshifts and spectral parameters of time-integrated spectrum up to October 2011. However more than half of their spectral parameters determined by not the Band model (Band et al. 1993), but by the Cut-off power law model (Pendleton et al. 1997) because of the lack of number of high energy photon and/or the limited energy range of detectors, which tends to overestimate spectral peak energies (Kaneko et al. 2006; Krimm et al. 2009; Tsutsui et al. 2010). Using these biased data, the difference between subclasses is smoothed out with their systematic errors (Tsutsui et al. 2011). Therefore we do not deal with these data from the beginning in this paper. Then, we use only 44 LGRBs with known redshifts, spectral parameters determined by the Band model, observer frame 1-second peak fluxes, fluences, and $64 \mathrm{msec}$ resolution light curves.

For the GRBs detected by GBM/Fermi, the spectral analysis is performed with the software package RMFIT ${ }^{2}$ (version 3.3rc8) and the GBM Response Matrices v1.8. We analyze the time-integrated spectrum using CSPEC data, from $8 \mathrm{keV}$ to $40 \mathrm{MeV}$ with $1.024 \mathrm{sec}$ temporal resolution, following the guidance of the RMFIT tutorial ${ }^{3}$.

For other GRBs, there are spectral parameters of individual GRBs reported by different observation teams and different authors, so that we choose the data fitted by the Band model with the smallest uncertainties. Because we did not know the systematic difference between $E_{\mathrm{p}}$ estimated with the Band model and $E_{\mathrm{p}}$ with the CPL model in our previous studies, the data in this paper is not exactly the same as the data in Yonetoku et al. (2010) and Tsutsui et al. (2011).

2 http://fermi.gsfc.nasa.gov/ssc/data/analysis/

3 http://fermi.gsfc.nasa.gov/ssc/data/analysis/user/vc_rmfittutorial.pdf http://gcn.gsfc.nasa.gov/konus_grbs.html
The KONUS team often reports not 1 sec peak fluxes but $64,128,256$ msec fluxes, so that we convert these peak fluxes to $1 \mathrm{sec}$ peak fluxes with $64 \mathrm{msec}$ light curves obtained from the archive of all the GCN/KONUS Notices and Light Curves ${ }^{4}$ (Yonetoku et al. 2010).

For all data reported with errors at $90 \%$ confidence level, we convert it to errors at $68 \%$ one by multiplying $1 / 1.645$. In table 6 , we summarize redshifts, spectral parameters, observed 1-second fluxes and fluences with detector's energy band and their references of 44 LGRBs.

From these parameters, bolometric 1-second luminosities $\left(L_{\mathrm{p}, 1 \mathrm{~s}}^{\mathrm{obs}}\right)$ and isotropic equivalent energies $\left(E_{\text {iso }}\right)$ between $1-10,000 \mathrm{keV}$ in GRB rest frame are calculated by following equations:

$$
\begin{gathered}
L_{\mathrm{p}, 1}^{\mathrm{obs}}=4 \pi d_{\mathrm{L}}^{2} P_{\mathrm{p}, \mathrm{obs}} \times \frac{\int_{1 /(1+z)}^{10,000 /(1+z)} E \times N(E) d E}{\int_{E_{\min }}^{E_{\max }} N(E) d E}\left(\mathrm{erg} \mathrm{s}^{-1}\right), \\
E_{\mathrm{iso}}=\frac{4 \pi d_{\mathrm{L}}^{2}}{1+z} S_{\mathrm{obs}} \times \frac{\int_{1 /(1+z)}^{10,000 /(1+z)} E \times N(E) d E}{\int_{E_{\min }}^{E_{\max }} E \times N(E) d E} \\
(\mathrm{erg}) .
\end{gathered}
$$

where $N(E)$ is the Band model in units of photons $\mathrm{cm}^{-2} \mathrm{~s}^{-1} \mathrm{keV}^{-1}$ (Band et al. 1993) and $d_{\mathrm{L}}$ is the luminosity distance in units of $\mathrm{cm}$. We should note that $L_{\mathrm{p}, 1}^{\mathrm{obs}}$ 's are calculated within 1-second in observer frame, which must be converted to rest $\tau$-second peak luminosities $L_{\mathrm{p}, \tau}^{\text {rest's }}$ (Yonetoku et al. 2010; Tsutsui et al. 2011). In this paper, we adopt $\tau=2.752$ second for all GRBs. 3).

There is another parameter characterizing a time scale of GRBs. Firmani et al. (2006) used the high signal time $T_{0.45}$ to improve the $E_{\mathrm{p}}-L_{\mathrm{p}}$ correlation. Here we adopt another time scale, the luminosity time $T_{\mathrm{L}}$, first used by Tsutsui et al. (2009) defined as,

$$
T_{\mathrm{L}} \equiv \frac{E_{\text {iso }}}{L_{\mathrm{p}}},
$$

where an error of $T_{\mathrm{L}}$ is calculated from the error propagation formula without the cross-term.

In this paper, we analyze light curves of LGRBs to identify subclasses of LGRBs. For secure analysis of light curves we use only the bursts whose background fluctuation - peak counts ratio are less than 0.1. This reduces the number of GRBs from 44 to 36 . Then we analyze 36 bursts in following sections. In table 7 , we summarize the intrinsic properties of the 36 GRBs.

\section{Fundamental Plane of Long Gamma-Ray Bursts}

The time scales which improve the $E_{\mathrm{p}}-L_{\mathrm{p}}$ correlation have been studied in the past. Some obtained positive results (Firmani et al. 2006; Tsutsui et al. 2009) and the others negative results (Rossi et al. 2008; Collazzi \& Schaefer 
2008). There are several reasons that the previous studies could not establish the tight correlation between the spectral peak energy, the time scale, and the peak luminosity : systematic errors of peak energies estimated by the CPL model, systematic errors of peak luminosities estimated in 1 seconds of observer, and contaminations of short GRBs with extended emission or other outliers. They have all disturbed the estimation of the best fit function and their dispersion so that such tight correlations have never been reproduced without the studies of the systematics as mentioned above (Yonetoku et al. 2010; Tsutsui et al. 2010; Tsutsui et al. 2011).

Here we briefly describe the method developed in Tsutsui et al. (2011). We assume a linear correlation between $E_{\mathrm{p}}, T_{\mathrm{L}}$, and $L_{\mathrm{p}}$ in a logarithmic form as:

$$
\begin{aligned}
\log L_{\mathrm{p}}\left(E_{\mathrm{p}}, T_{\mathrm{L}}\right)=A+ & B \log \left(\frac{E_{\mathrm{p}}}{10^{2.71} \mathrm{keV}}\right) \\
+ & C \log \left(\frac{T_{\mathrm{L}}}{10^{0.86} \mathrm{sec}}\right),
\end{aligned}
$$

where $A, B$, and $C$ are free parameters of the model. If we adopt $C=0$, and $C=-1$, this is identical with the $E_{\mathrm{p}}-L_{\mathrm{p}}$ and $E_{\mathrm{p}}-E_{\text {iso }}$ correlations, respectively.

The corresponding $\chi^{2}$ function is given by :

$$
\begin{aligned}
& \chi^{2}(A, B, C, \tau)=\sum_{i} z_{i}^{2}, \\
& z_{i}=\frac{\log L_{\mathrm{p}, \tau i}^{\text {rest }}-\log L_{\mathrm{p}}\left(E_{\mathrm{p}, i}, T_{\mathrm{L}, i}\right)}{\sqrt{(1+2 C) \sigma_{\log L_{\mathrm{p}, i}}^{2}+B^{2} \sigma_{\log E_{\mathrm{p}, i}}^{2}+C^{2} \sigma_{\log T_{\mathrm{L}, i}}^{2}+\sigma_{\text {int }}^{2}}}
\end{aligned}
$$

The factor $2 C$ in front of $\sigma_{\log L_{\mathrm{p}}}$ comes from the fact that the definition of $T_{\mathrm{L}}$ includes $L_{\mathrm{p}}$.

Because the $\chi^{2}$ value strongly depends on the existence of outliers which deviate from the Gaussian distribution, we first optimize the Lorentzian merit function, given by

$$
M(A, B, C, \tau)=\sum_{i} \ln \left(1+\frac{1}{2} z_{i}^{2}\right),
$$

with $\sigma_{\text {int }}=0$. Because the Lorentzian merit function is less affected by outliers which do not follow Gaussian distribution, we use the parameters optimizing the Lorentzian merit as the tentative values to eliminate outliers and to estimate robust $\sigma_{\text {int }}$ value.

To estimate robust $\sigma_{\text {int }}$, we use the robust standard deviation given by,

$$
\sigma_{\mathrm{RSD}} \equiv \frac{\operatorname{median}\left[\left|\log L_{\mathrm{p}, \tau i}^{\mathrm{rest}}-\log L_{\mathrm{p}}\left(E_{\mathrm{p}, i}, T_{\mathrm{L}, i}\right)\right|\right]}{0.6745} .
$$

and then we obtain robust $\sigma_{\text {int }}$ as follows :

$$
\begin{aligned}
& \sigma_{\text {int }}^{2}=\sigma_{R S D}^{2} \\
& -\frac{1}{N} \sum_{i}^{N}\left\{(1+2 C) \sigma_{\log L_{\mathrm{p}, i}}^{2}+B^{2} \sigma_{\log E_{\mathrm{p}, i}}^{2}+C^{2} \sigma_{\log T_{\mathrm{L}, i}}^{2}\right\} .
\end{aligned}
$$

Now that we have tentative sets of parameters and $\sigma_{\text {int }}$, we can compute, for each sample, $t=\left|z_{i}\right|$ and the twotailed $\mathrm{P}$-value from the $t$ distribution with $(N-3)$ degrees of freedom. We adopt a threshold probability as $P_{\text {th }}=$ $Q / N$ for all GRBs, and the GRBs which have P-value smaller than $P_{\text {th }}$ are regarded as outliers and eliminated from the following chi square analysis. Here Q is arbitrary number less than 1 . If it is not mentioned we always use $Q=0.2$ in this paper. Therefore we now have data sets without outliers and $\sigma_{\text {int }}$, and we can perform the ordinary chi square analysis to estimate the best fit parameters and their uncertainties.

First we apply this method to our updated sample, both the whole sample and the platinum sample with $\sigma_{\mathrm{E}_{\mathrm{p}}} / E_{\mathrm{p}}<0.1$. In Tsutsui et al. (2011), using only the platinum sample we obtained the very tight correlation with six outliers. The reason for only the platinum sample is to distinguish possible outliers or subclasses from the observational errors. As a result in Tsutsui et al. (2011) we suggested that there might be three subclasses in LGRBs. In figure 1 , we plot the $E_{\mathrm{p}}-T_{\mathrm{L}}-L_{\mathrm{p}}$ diagram obtained by the present platinum sample. The platinum events consistent with the $E_{\mathrm{p}}-T_{\mathrm{L}}-L_{\mathrm{p}}$ correlation, that is, on plane events, are marked with red circles, and outliers with green triangles. In table 1, we summarize the best fit parameters, fraction of outliers $\left(N_{\text {out }} / N\right)$, intrinsic dispersion $\left(\sigma_{\text {int }}\right)$ and reduced chi square $\left(\chi_{\nu}^{2}\right)$ both for the whole sample and the platinum sample. As table 1 shows, the best fit values of parameters, $\sigma_{\text {int }}$, and the fraction of outliers $\left(N_{\text {out }} / N\right)$ depend on the sample. We see that $\sigma_{\text {int }}$ for the whole sample is 2.6 times larger than that for the platinum sample. While the outliers exist for both samples so that the distributions are not Gaussian for both samples. This fact suggests that strong non-gaussianity, perhaps bimodality of the scatters around the best fit function exists. As figure 1 indicates, there might be a subclass of GRBs (green triangles in the upper left corner) which might be another correlation nearly parallel to the $E_{\mathrm{p}}-T_{\mathrm{L}}-L_{\mathrm{p}}$ correlation. This situation is similar to PoPI and PoPII Cepheid variable correlations that are parallel due to the difference of metallicity. In practice, however, it is sometime difficult to remove all the outliers as shown in Monte Carlo simulations in Appendix A in Tsutsui et al. (2011) even if we use the outlier detection technique based on robust statistics - This might be the reason for the best fit parameters and $\sigma_{\text {int }}$ for platinum sample in table 1 being different from the ones in Tsutsui et al. (2011) although the tightness of the correlation is unchanged. This fact suggests that there is a large fraction of outliers close to the best fit function. If we can remove these outliers using other observational properties in advance, the Fundamental Plane of LGRBs will be more statistically significant and robust. The purpose of this paper is to find what is this other observational property like the metallicity in PoPI and PoPII Cepheid variable correlations.

In the following sections, we introduce a new parameter of LGRBs, the absolute deviation from their constant luminosity $(A D C L)$. We show that dividing LGRBs according to $A D C L$-value significantly improves both of the $E_{\mathrm{p}}-L_{\mathrm{p}}$ correlation and the Fundamental Plane of GRBs. 
That is, we show that ADCL is a new observational property what we have been seeking for.

Table 1. Fitting results for our new sample.

by ourselves. The most popular duration of GRBs is $T_{90}$ defined by Kouveliotou et al. (1993). $T_{90}$ is the time during which the cumulative counts increase from $5 \%$ to $95 \%$. However, we find that $T_{90}$ tends to lose weak long tails of bursts which are essential to classify the bursts. Thus, we yse $T_{98}$ to define $A D C L$ instead of $T_{90}$.

\begin{tabular}{|c|c|c|c|c|}
\hline sample & best fit & $\sigma_{\text {int }}$ & $N_{\text {out }} / N$ & $\chi_{\nu}^{2}$ \\
\hline all & $(52.63,1.63,-0.08)$ & 0.13 & $8 / 36$ & $20.9 / 26$ \\
\hline platinum & $(52.52,1.86,0.31)$ & 0.05 & $11 / 23$ & $3.81 / 10$ \\
\hline
\end{tabular}

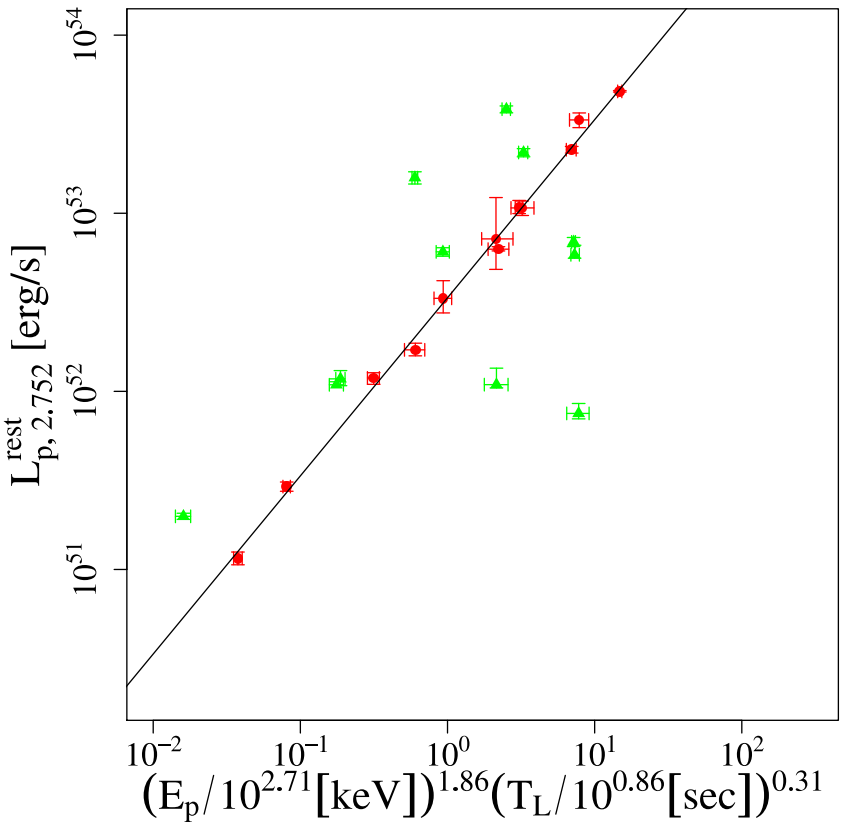

Fig. 1. The $E_{\mathrm{p}}-T_{\mathrm{L}}-L_{\mathrm{p}}$ diagram of updated platinum sample. The green triangles represent outliers.

\section{Absolute Deviation from Constant Luminosity (ADCL)}

To quantify the absolute deviation from their constant luminosity $(A D C L)$, we analyze $64 \mathrm{msec}$ resolution light curves of BATSE, KONUS, Swift, and GBM. We summarize the energy band of each detector in table 2 .

Table 2. The energy band of light curves for each detector

\begin{tabular}{|c|c|c|c|}
\hline BATSE & KONUS & Swift & GBM \\
\hline $20-1000 \mathrm{keV}$ & $50-200 \mathrm{keV}$ & $15-150 \mathrm{keV}$ & $8-1000 \mathrm{keV}$ \\
\hline
\end{tabular}

We determine the background intervals before and after bursts for BATSE, Swift, and GBM light curves and make linear fit of background using least square method. For KONUS light curves, there are no pubic data before bursts so that we use the background intervals only after bursts. Then we subtract the backgrounds from $64 \mathrm{msec}$ light curves interpolating or extrapolating the fit across the whole duration of bursts. From these background subtracted light curves, we determine the duration of bursts

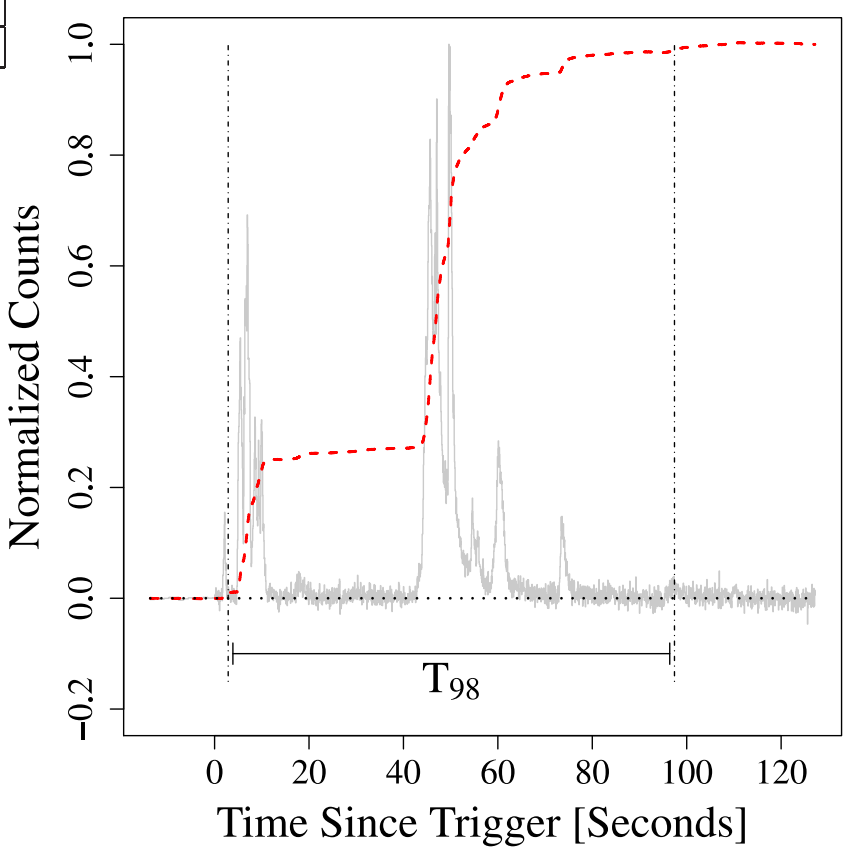

Fig. 2. An example of the cumulative light curve of GRB 990506. The cumulative light curve is plotted with a red dashed line, and the ordinary light curve with a gray solid line. The vertical lines show the start and end time of $T_{98}$. The interval within $T_{98}$ is indicated by an arrow.

Because the observed distribution of GRBs spans the wide range of redshift, from 0.0085 to 8.2 up to present, and the cosmological time dilation changes the time scales of bursts by factor $(1+z)$, the measurement of temporal properties of bursts must be robust to the change of time resolution of light curves. Besides, recent results from hydrodynamical simulations of jet imply that the long term variability of GRBs come from the activity of the central engine, while the short term variability from the propagation of jet (Morsony et al. 2010) and we should decompose long and short term variability in some way. For these reasons, we use cumulative light curves to quantify the difference of bursts which are much simpler than ordinary light curves (McBreen et al. 2002; Varga et al. 2005). Figure 2 shows an example of the cumulative light curve of GRB 990506. The cumulative light curve normalized with total counts is plotted with a red dashed line, and the ordinary light curve normalized with peak counts with a gray solid line, respectively. The short scale structure in the ordinary light curve is canceled out in the cumulative light curve, and then the analysis of cumulative light curves is much robust to a cosmological time dilation and choice of the time resolution.

To compare pulse shapes of bursts with various dura- 
tions, we normalize the time scale of bursts with $T_{98}$ and then all bursts have normalized cumulative counts $C_{i}^{\text {norm }}$ from 0.01 to 0.99 , and normalized time $t_{i}^{\text {norm }}$ from 0 to 1 . Figure 3 shows normalized cumulative light curves of the platinum samples in table 7 . On-plane events are plotted with red lines, outliers with green lines in figure 3 . The black dotted line is the line of the virtual source with the constant luminosity. The brighter and dimmer outliers deviate from the constant luminosity line, while many of on-plane events cluster around the constant luminosity line.

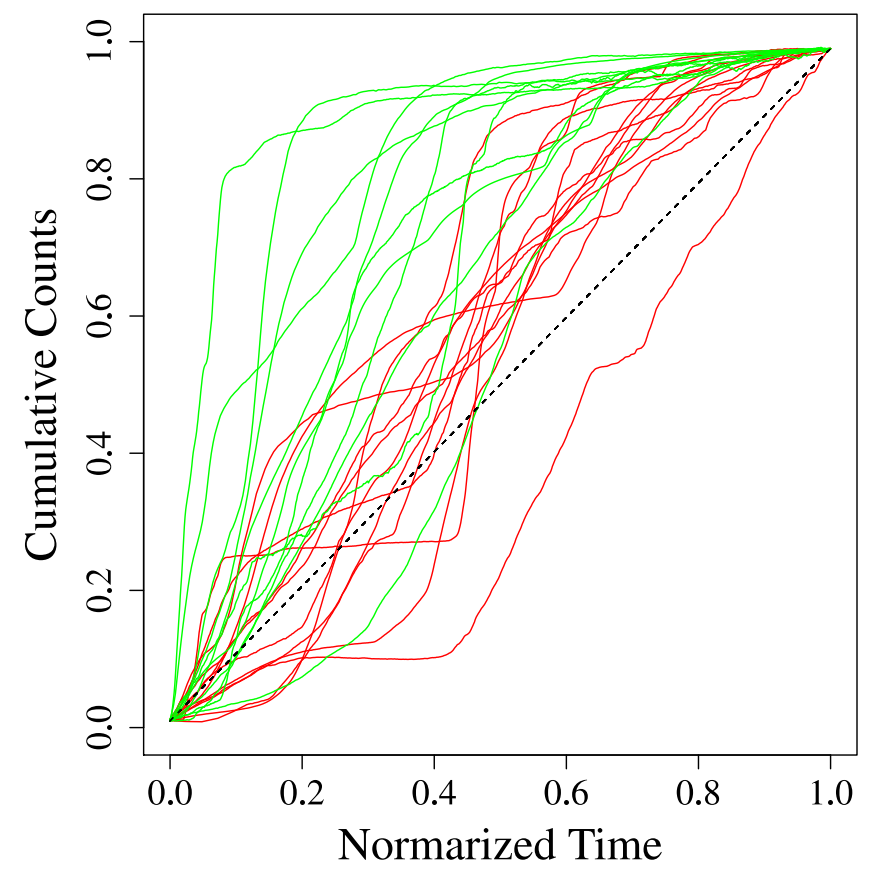

Fig. 3. The normalized cumulative light curves for the platinum samples. On-plane events are plotted with red solid lines, outliers with green solid lines. The black dotted line is the line of the virtual source with the constant luminosity.

To quantify these difference, we introduce a new parameter of GRBs : the absolute deviation from their constant luminosity $(A D C L)$. We define the absolute deviation from their constant luminosity :

$$
A D C L=\sum_{i=1}^{N_{\text {bin }}} \frac{\left|C_{i}^{\text {norm }}-0.01-0.98 \times t_{i}^{\text {norm }}\right|}{N_{\text {bin }}}
$$

where the number of the bin $\left(N_{\text {bin }}\right)$ is different from burst to burst. Figure 4 shows the distribution of $A D C L$. The distribution of $A D C L$ in figure 4 indicates that there is a gap around $\log (A D C L) \sim-0.75(A D C L \sim 0.17)$. From this result, we divide the LGRBs into two subclasses : one is small- $A D C L$ events with $A D C L<0.17$ and the other is long tailed ones with $A D C L>0.17$. Figure 5 shows examples of cumulative light curves of small- $A D C L$ (left) and long tailed (right) events, respectively. Although it is difficult to quantify the statistical significance of the existence of the gap, we will show that the gap becomes more clear when we study the relationship between the $A D C L$ and

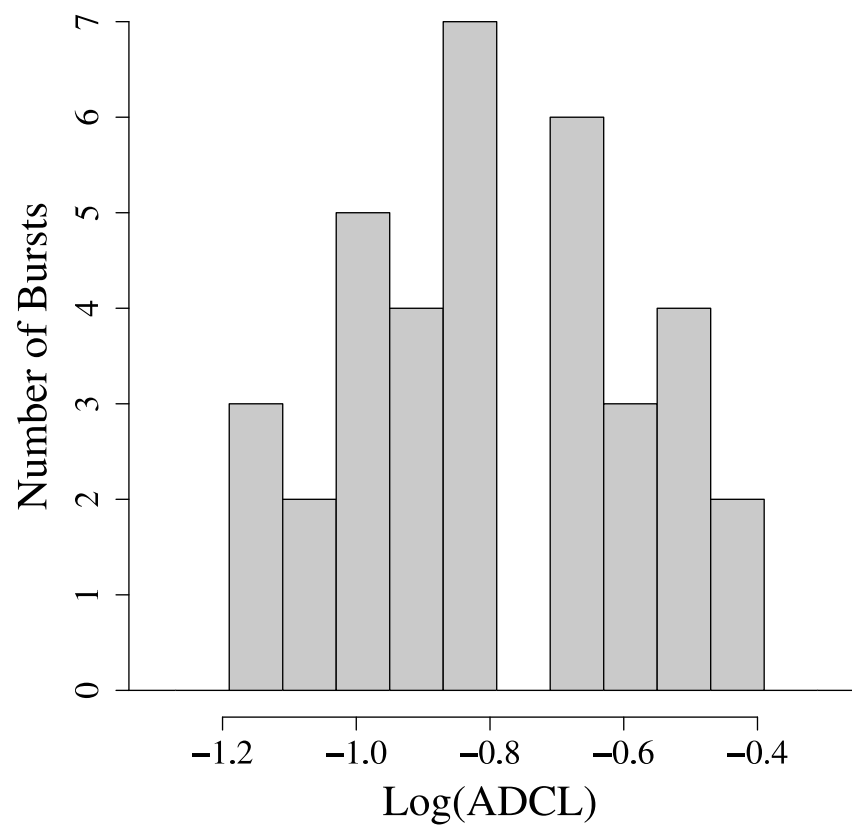

Fig. 4. The distribution of $A D C L$. There is a gap around $\log (A D C L) \sim-0.75$.

residual from the Fundamental Plane of LGRBs. This is very similar to the discovery of the short - hard and long soft classification by Kouveliotou et al. (1993). Only from $T_{90}$, the long and short classification is not so clear that they added the spectral hardness. Then they could identify the bimodal distribution of the long-soft and shorthard GRBs clearer. In our case, the residual from the EpTL-Lp correlation corresponds to the hardness. In Figure 8. we can identify two suclasses (blue squares and red circles) in the ADCL-residual plane.

In table 7 , we summarize the intrinsic properties and $A D C L$ of the 36 GRBs.

\subsection{Type I Fundamental plane}

In Tsutsui et al. (2011) and §-3, we have found that there are probably three classes of LGRBs : on-plane events, brighter outliers, and dimmer outliers. In section 4, we introduced a new parameter $A D C L$ and found that the most of on-plane events have smaller $A D C L$, while most of the brighter and dimmer outliers have relatively larger $A D C L$. In this section, we derive the best fit function and dispersion of the $E_{\mathrm{p}}-T_{\mathrm{L}}-L_{\mathrm{p}}$ correlation for small$A D C L$ and large- $A D C L$ GRBs separately, using the same method in section 3 .

We used only the platinum data with $\sigma_{E_{\mathrm{p}}} / E_{\mathrm{p}}<0.10$ in Tsutsui et al. (2011) and section 3 to avoid the contamination of outliers and subclasses because there are no prior knowledge about outliers and subclasses of LGRBs. Now ADCL is a candidate for another observational property to distinguish outliers and subclass of LGRBs. Using ADCL we might eliminate brighter and dimmer outliers in advance. Therefore we do not need to restrict the sample to the platinum one from now on. The platinum sample was needed only to find ADCL so that we will use 
the whole sample in the following analysis although larger observational errors compared with the platinum sample exist.

First, we apply the outlier elimination technique to the small- $A D C L$ GRBs with $A D C L<0.17$ and find the $E_{\mathrm{p}}-$ $T_{\mathrm{L}}-L_{\mathrm{p}}$ correlation of small- $A D C L$ GRBs becomes tightest around $\tau=2.752$ seconds. The best fit relation is given by,

$$
\begin{aligned}
L_{\mathrm{p}}=10^{52.53 \pm 0.01} & \left(\frac{E_{\mathrm{p}}}{10^{2.71} \mathrm{keV}}\right)^{1.84 \pm 0.03} \\
& \times\left(\frac{T_{\mathrm{L}}}{10^{0.86} \mathrm{sec}}\right)^{0.29 \pm 0.08},
\end{aligned}
$$

with $\chi_{\nu}^{2}=10.93 / 14$ and $\sigma_{\text {int }}=0$. The power-law index of $T_{L}$ in equation. (11) is different from that of the equation. (12) of Tsutsui et al., (2011). This is because there were some contamination of long tailed GRBs for the lack of population of LGRBs. Hereafter we refer to the equation (11) as the Type I Fundamental Plane.

In figure 6 , we plot the data of the small- $A D C L$ events with $A D C L<0.17$ and the best-fit function of the Type I Fundamental Plane. The small- $A D C L$ events used to derive the best-fit function were marked with red circles and the events regarded as outliers with green triangles. The solid line represents the best-fit function of the Type I Fundamental Plane.

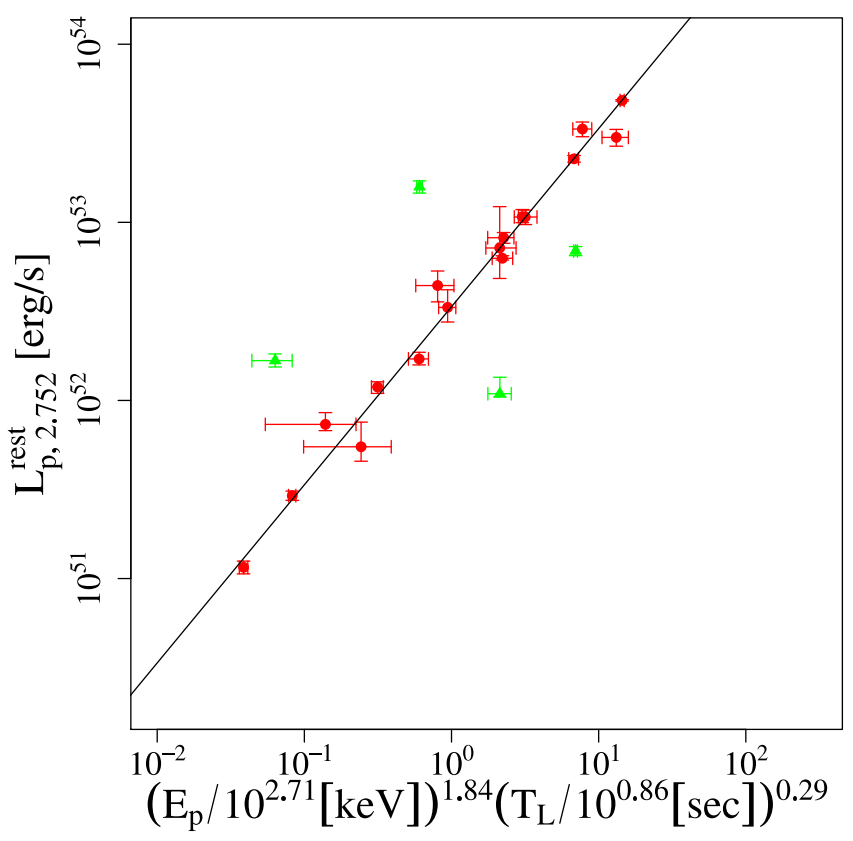

Fig. 6. The Type I Fundamental Plane of small- $A D C L$ events $(A D C L<0.17)$. The red circles represent the small-ADCL event used to derive the Type I Fundamental Plane and the green triangles represent outliers eliminated from chi square analysis. The solid line represents the equation (11).

\subsection{Type II Fundamental Plane}

We find that large- $A D C L$ GRBs form the other Fundamental Plane which is almost parallel to Type
I Fundamental Plane but slightly brighter than it. Furthermore, we find that all GRBs which have large $T_{\mathrm{L}}>12 \mathrm{sec}$ deviate from other events, and we eliminate them from following analysis. Then we apply the outlier elimination technique to the long tailed events with $T_{\mathrm{L}}<12 \mathrm{sec}$ and estimate the best fit parameters and resolution time scale of long tailed events. We find the $E_{\mathrm{p}}-$ $T_{\mathrm{L}}-L_{\mathrm{p}}$ correlation of the long tailed events also becomes tightest around 2.7 seconds. Adopting 2.752 seconds as the resolution time scales of large- $A D C L$ events, we obtain

$$
\begin{aligned}
L_{\mathrm{p}}=10^{52.98 \pm 0.080} & \left(\frac{E_{\mathrm{p}}}{10^{2.71} \mathrm{keV}}\right)^{1.82 \pm 0.093} \\
& \times\left(\frac{T_{\mathrm{L}}}{10^{0.86} \mathrm{sec}}\right)^{0.85 \pm 0.26},
\end{aligned}
$$

with $\chi_{\nu}^{2}=7.58 / 8$ and $\sigma_{\text {int }}=0$ for long tailed GRBs. Hereafter we refer to the equation (12) as the Type II Fundamental Plane. The Type II Fundamental Plane is about 2.5 times brighter than the Type I Fundamental Plane.

In figure 7 , we plot the data of large- $A D C L$ events and the best-fit function of the Type II Fundamental Plane. The events used to derive the best-fit function were marked with blue squares and the events regarded as outliers with green triangles. The solid line represents the best-fit function of the Type II Fundamental Plane.

Figure 8 shows the relationship between residual from the Type I fundamental plane and $A D C L$ of all events. Each symbol represents same as figure 6 and 7. Dimmer outliers apparently have intermediate $A D C L$ value. Figure 8 might imply that there are some other classes of LGRBs.

In section 3 we showed that the $E_{\mathrm{p}}-T_{\mathrm{L}}-L_{\mathrm{p}}$ correlation for whole sample has significant intrinsic dispersion $\left(\sigma_{\text {int }}=0.13\right.$ for whole sample while 0.05 for platinum sample. see Table. 1). If we divide the whole sample into two subclasses from their $A D C L$ values, $\sigma_{\text {int }}=0$ as shown in this chapter. Thus we can conclude that the $E_{\mathrm{p}}-T_{\mathrm{L}}-L_{\mathrm{p}}$ correlation is certainly improved by introducing $A D C L$.

\section{Discussion}

In this section, we would like to show the validity of the $E_{\mathrm{p}}-T_{\mathrm{L}}-L_{\mathrm{p}}$ correlation. First one might ask that $T_{\mathrm{L}}$ might introduce spurious correlation because $T_{\mathrm{L}}$ includes $L_{\mathrm{p}}$ in its definition. We checked this by assuming the relation as $\log L_{\mathrm{p}}\left(E_{\mathrm{p}}, E_{\text {iso }}\right)=A^{\prime}+B^{\prime} \log \left(E_{\mathrm{p}} / 10^{2.71} \mathrm{keV}\right)+$ $C^{\prime} \log \left(E_{\text {iso }} / 10^{53.40} \mathrm{sec}\right)$. We determined $A^{\prime}, B^{\prime}$ and $C^{\prime}$ using the same data and the same method. If there is no spurious correlation, we should obtain $B^{\prime}=B /(1+C)$ and $C^{\prime}=C /(1+C)$ where $\mathrm{A}, \mathrm{B}$ and $\mathrm{C}$ are defined in Eq. (4). We obtained that $B^{\prime}=1.42, C^{\prime}=0.23$ with $\chi_{\nu}^{2}=10.93 / 14 . \quad B=1.84, C=0.29$ so that $B /(1+C)=$ $1.42, C /(1+C)=0.22$ for small- $A D C L$ sample and that $B^{\prime}=0.98, C^{\prime}=0.46$ with $\chi_{\nu}^{2}=7.59 / 9 . B=1.82, C=0.85$ so that $B /(1+C)=0.98, C /(1+C)=0.46$ for large ADCL 


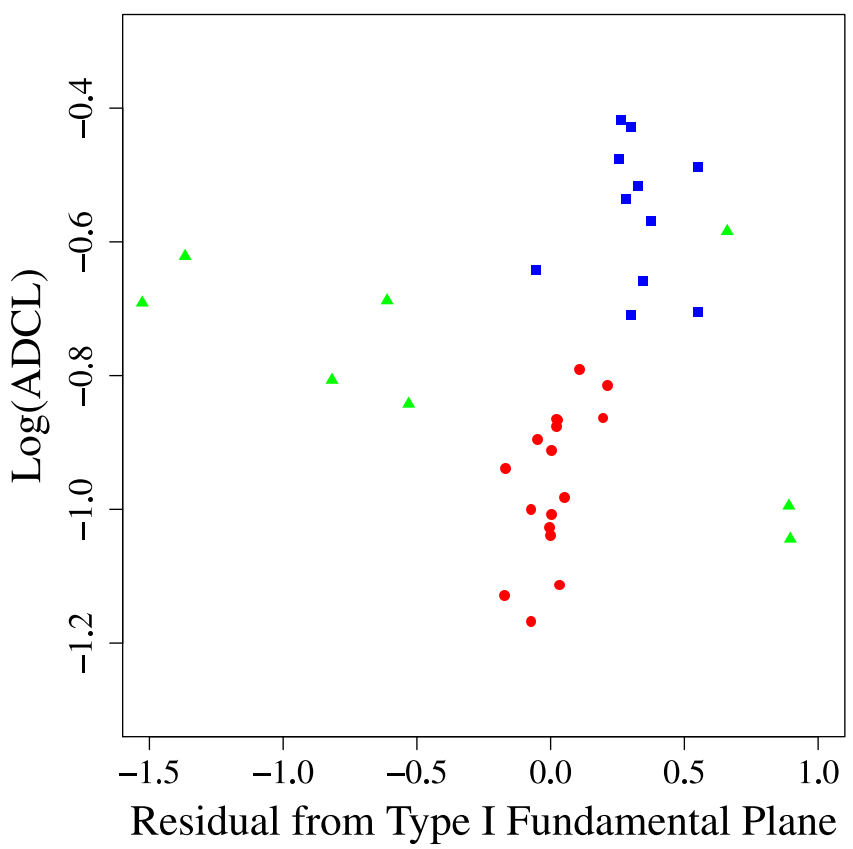

Fig. 8. The relationship between the residual from Type I fundamental plane and $A D C L$ of all events. Each symbol represents same as figure 6 and 7 .

sample. These values show that the spurious correlation is very small even if it exists. Therefore which one should be used is a matter of taste.

Next we compare the $E_{\mathrm{p}}-T_{\mathrm{L}}-L_{\mathrm{p}}$ correlation with $E_{\mathrm{p}}$ $L_{\mathrm{p}}$ correlation for each subsample with the same statistical method in section 3 . We first derive the $E_{\mathrm{p}}-L_{\mathrm{p}}$ correlation with the whole sample as discussed in section 3. In table 3 we summarize the best fit parameters, fraction of outliers $\left(N_{\text {out }} / N\right)$, intrinsic dispersion $\left(\sigma_{\text {int }}\right)$ and reduced chi square $\left(\chi_{\nu}^{2}\right)$ both for the whole sample and platinum sample. As table 1 and 3 indicate the $E_{\mathrm{p}}-L_{\mathrm{p}}$ correlation has the larger systematic dispersion than the $E_{\mathrm{p}}-T_{\mathrm{L}}-L_{\mathrm{p}}$ correlation when we use the whole sample. the whole sample.

Secondly we divide the GRBs into two groups by $A D C L$, and derive the $E_{\mathrm{p}}-L_{\mathrm{p}}$ correlation for each sample. Here we should note without the $E_{\mathrm{p}}-T_{\mathrm{L}}-L_{\mathrm{p}}$ correlation it was not possible to find ADCL and to recognize the existence of the subclasses. However now we know that two subclasses exist so that we check the $E_{\mathrm{p}}-L_{\mathrm{p}}$ correlation for each subclass. In table 4 and 5 , we summarize the best fit parameters, fraction of outliers $\left(N_{\text {out }} / N\right)$, intrinsic dispersion $\left(\sigma_{\text {int }}\right)$ and reduced chi square $\left(\chi_{\nu}^{2}\right)$ for each correlation and each subclass. As table 4 and 5 indicate, the difference in parameters of the $E_{\mathrm{p}}-L_{\mathrm{p}}$ correlation between small- $A D C L$ events and large- $A D C L$ (long tailed) events is smaller than that of the $E_{\mathrm{p}}-T_{\mathrm{L}}-L_{\mathrm{p}}$ correlation. Therefore we can not distinguish them even if we use our outlier rejection technique since the difference of the amplitude $\mathrm{A}$ is almost the same $(\sim 0.2$ in logarithm $E_{\mathrm{p}}-L_{\mathrm{p}}$ correlation while $\sim 0.45$ for $E_{\mathrm{p}}-T_{\mathrm{L}}-L_{\mathrm{p}}$ correlation) . Therefore $E_{\mathrm{p}}-T_{\mathrm{L}}-L_{\mathrm{p}}$ correlation is needed to distinguish two subclasses. In case of small- $A D C L$ events, the $E_{\mathrm{p}}-L_{\mathrm{p}}$ correlation has slightly larger dispersion than the $E_{\mathrm{p}}-T_{\mathrm{L}}-$ $L_{\mathrm{p}}$ correlation, although the $E_{\mathrm{p}}-L_{\mathrm{p}}$ correlation has one more outlier. Taking into these facts, we can insist that the $E_{\mathrm{p}}-T_{\mathrm{L}}-L_{\mathrm{p}}$ correlation is surely more statistically significant than $E_{\mathrm{p}}-L_{\mathrm{p}}$ correlation for small- $A D C L$ events. In case of long tailed events, however, it is difficult to say which one is more significant. The $E_{\mathrm{p}}-L_{\mathrm{p}}$ correlation has two more outliers, but smaller dispersion than the $E_{\mathrm{p}}$ $T_{\mathrm{L}}-L_{\mathrm{p}}$ correlation. To show the validity of the $E_{\mathrm{p}}-T_{\mathrm{L}}-L_{\mathrm{p}}$ correlation for long tailed events, we need larger number of data.

Table 3. Fitting results for the $E_{\mathrm{p}}-L_{\mathrm{p}}$ correlation with the whole sample.

\begin{tabular}{|c|c|c|c|c|}
\hline sample & best fit & $\sigma_{\text {int }}$ & $N_{\text {out }} / N$ & $\chi_{\nu}^{2}$ \\
\hline all & $(52.64,1.62)$ & 0.18 & $6 / 36$ & $27.6 / 28$ \\
\hline platinum & $(52.57,1.71)$ & 0.15 & $6 / 23$ & $12.9 / 15$ \\
\hline
\end{tabular}

\section{Summary}

In this paper we defined $A D C L$ which characterizes the cumulative light curve of the prompt emission of long GRBs. We divided the events into two groups according to the value of $A D C L$. Then we derived Fundamental Planes for the two groups separately and it was found that they are tighter and statistically more significant than one derived from the whole sample. Although we introduced the new parameter $A D C L$ to reduce the dispersion of the Fundamental Plane, we found dividing the events into two groups also improves the $E_{\mathrm{p}}-L_{\mathrm{p}}$ correlation. This fact supports the validity of introducing $A D C L$. These tighter relations would be helpful to use them as standard candles to probe cosmological expansion history. Also our analysis may imply the existence of subclasses of long GRBs classified by $A D C L$.

The discovery of these possible subclasses of GRBs reminds us of the discovery of the two separate subclasses of Cepheids by Baade. After Baade's discovery, the accuracy of the distance measurement by the Period-Luminosity relation of Cepheids was drastically improved. We can expect that the distance measurement by the $E_{\mathrm{p}}-T_{\mathrm{L}}-L_{\mathrm{p}}$ relation of GRBs will also be improved, and we are studying the distance measurement by the Type I Fundamental Plane of GRBs in near future.

\section{Acknowledgments}

This work is supported in part by the Grant-in-Aid from the Ministry of Education, Culture, Sports, Science and Technology (MEXT) of Japan, No.23540305 (TN), No.20674002 (DY), No.23740179 (KT), and by the Grantin-Aid for the global COE program The Next Generation of Physics, Spun from Universality and Emergence at Kyoto University. 
Table 4. Fitting results for small- $A D C L$ events.

\begin{tabular}{|c|c|c|c|c|}
\hline correlation & best fit & $N_{\text {out }} / N$ & $\sigma_{\text {int }}$ & $\chi_{\nu}^{2}$ \\
\hline$E_{\mathrm{p}}-L_{\mathrm{p}}$ & $(52.54,1.81)$ & $5 / 21$ & 0 & $15.55 / 14$ \\
\hline$E_{\mathrm{p}}-T_{\mathrm{L}}-L_{\mathrm{p}}$ & $(52.53,1,84,0.29)$ & $4 / 21$ & 0 & $10.93 / 14$ \\
\hline
\end{tabular}

Table 5. Fitting results for long tailed events.

\begin{tabular}{|c|c|c|c|c|}
\hline correlation & best fit & $N_{\text {out }} / \mathrm{N}$ & $\sigma_{\text {int }}$ & $\chi_{\nu}^{2}$ \\
\hline$E_{\mathrm{p}}-L_{\mathrm{p}}$ & $(52.73,1.78)$ & $6 / 15$ & 0 & $4.672 / 7$ \\
\hline$E_{\mathrm{p}}-T_{\mathrm{L}}-L_{\mathrm{p}}$ & $(52.98,1,82,0.85)$ & $4 / 15$ & 0 & $7.58 / 8$ \\
\hline
\end{tabular}

\section{References}

Amati, L., et al. 2002, Astronomy and Astrophysics, 390, 81

Band, D., et al. 1993, Astrophysical Journal, 413, 281

Band, D. L., \& Preece, R. D. 2005, The Astrophysical Journal, 627,319

Butler, N. R., Kocevski, D., Bloom, J. S., \& Curtis, J. L. 2007, The Astrophysical Journal, 671, 656

Cardone, V. F., Capozziello, S., \& Dainotti, M. G. 2009, Monthly Notices of the Royal Astronomical Society, 400, 775

Collazzi, A. C., \& Schaefer, B. E. 2008, The Astrophysical Journal, 688, 456

Diehl, S., \& Statler, T. S. 2005, The Astrophysical Journal, 633, L21

Djorgovski, S., \& Davis, M. 1987, Astrophysical Journal, 313, 59

Firmani, C., Ghisellini, G., Avila-Reese, V., \& Ghirlanda, G. 2006, Monthly Notices of the Royal Astronomical Society, 370,185

Ghirlanda, G., Ghisellini, G., \& Lazzati, D. 2004a, The Astrophysical Journal, 616, 331

Ghirlanda, G., Ghisellini, G., Lazzati, D., \& Firmani, C. 2004b, The Astrophysical Journal, 613, L13

Golenetskii, S., Aptekar, R., Mazets, E., Pal'Shin, V., Frederiks, D., \& Cline, T. 2006, GRB Coordinates Network, 5722, 1

—. 2007, GRB Coordinates Network, 6049, 1

- 2008a, GRB Coordinates Network, 7482, 1

Golenetskii, S., Aptekar, R., Mazets, E., Pal'Shin, V., Frederiks, D., Oleynik, P., Ulanov, M., Svinkin, D., \& Cline, T. 2008b, GRB Coordinates Network, 7995, 1

Golenetskii, S., Aptekar, R., Mazets, E., Pal'Shin, V., Frederiks, D., Oleynik, P., Svinkin, D., Ulanov, M., \& Cline, T. 2008c, GRB Coordinates Network, 8548, 1

Golenetskii, S., Aptekar, R., Mazets, E., Pal'Shin, V., Frederiks, D., Oleynik, P., Ulanov, M., Svinkin, D., \& Cline, T. 2009, GRB Coordinates Network, 9030, 1

—. 2011a, GRB Coordinates Network, 1197

Golenetskii, S., Aptekar, R., Frederiks, D., Mazets, E., Pal'Shin, V., Oleynik, P., Ulanov, M., Svinkin, D., \& Cline, T. 2011b, GRB Coordinates Network, 1200

—. 2011c, GRB Coordinates Network, 1216

Kaneko, Y., Preece, R., Briggs, M., Paciesas, W., Meegan, C., \& Band, D. 2006, The Astrophysical Journal Supplement Series, 166, 298

Kodama, Y., Yonetoku, D., Murakami, T., Tanabe, S., Tsutsui, R., \& Nakamura, T. 2008, Monthly Notices of the Royal Astronomical Society: Letters, 391, L1
Kouveliotou, C., Meegan, C. A., Fishman, G. J., Bhat, N. P., Briggs, M. S., Koshut, T. M., Paciesas, W. S., \& Pendleton, G. N. 1993, Astrophysical Journal, 413, L101

Krimm, H. A., et al. 2006, GAMMA-RAY BURSTS IN THE SWIFT ERA: Sixteenth Maryland Astrophysics Conference. AIP Conference Proceedings, 836, 145

-. 2009, The Astrophysical Journal, 704, 1405

Liang, E., \& Zhang, B. 2005, The Astrophysical Journal, 633, 611

Liang, N., Xiao, W. K., Liu, Y., \& Zhang, S. N. 2008, The Astrophysical Journal, 685, 354

McBreen, S., McBreen, B., Hanlon, L., \& Quilligan, F. 2002, Astronomy and Astrophysics, 393, L29

Merloni, A., Heinz, S., \& di Matteo, T. 2003, Monthly Notices of the Royal Astronomical Society, 345, 1057

Morsony, B. J., Lazzati, D., \& Begelman, M. C. 2010, The Astrophysical Journal, 723, 267

Nakar, E., \& Piran, T. 2005, Monthly Notices of the Royal Astronomical Society: Letters, 360, L73

Pendleton, G., et al. 1997, The Astrophysical Journal, 489, 175

Rossi, F., et al. 2008, Monthly Notices of the Royal Astronomical Society, 388, 1284

Sakamoto, T., et al. 2005, The Astrophysical Journal, 629, 311

Schaefer, B. E. 2007, The Astrophysical Journal, 660, 16

Schaeffer, R., Maurogordato, S., Cappi, A., \& Bernardeau, F. 1993, R.A.S. MONTHLY NOTICES V.263, 263, L21

Shahmoradi, A., \& Nemiroff, R. J. 2011, Monthly Notices of the Royal Astronomical Society, 411, 1843

Tsutsui, R., Nakamura, T., Yonetoku, D., Murakami, T., Kodama, Y., \& Takahashi, K. 2009, Journal of Cosmology and Astroparticle Physics, 08, 015

Tsutsui, R., Nakamura, T., Yonetoku, D., Murakami, T., Morihara, Y., \& Takahashi, K. 2011, Publications of the Astronomical Society of Japan, 63, 741

Tsutsui, R., Nakamura, T., Yonetoku, D., Murakami, T., \& Takahashi, K. 2010, eprint arXiv:1012.3009

Varga, B., Horváth, I., \& Balázs, L. G. 2005, Il Nuovo Cimento C, 28, 861

Yonetoku, D., Murakami, T., Nakamura, T., Yamazaki, R., Inoue, A. K., \& Ioka, K. 2004, The Astrophysical Journal, 609, 935

Yonetoku, D., Murakami, T., Tsutsui, R., Nakamura, T., Morihara, Y., \& Takahashi, K. 2010, Publications of the Astronomical Society of Japan, 62, 1495

Yu, B., Qi, S., \& Lu, T. 2009, The Astrophysical Journal Letters, 705, L15 

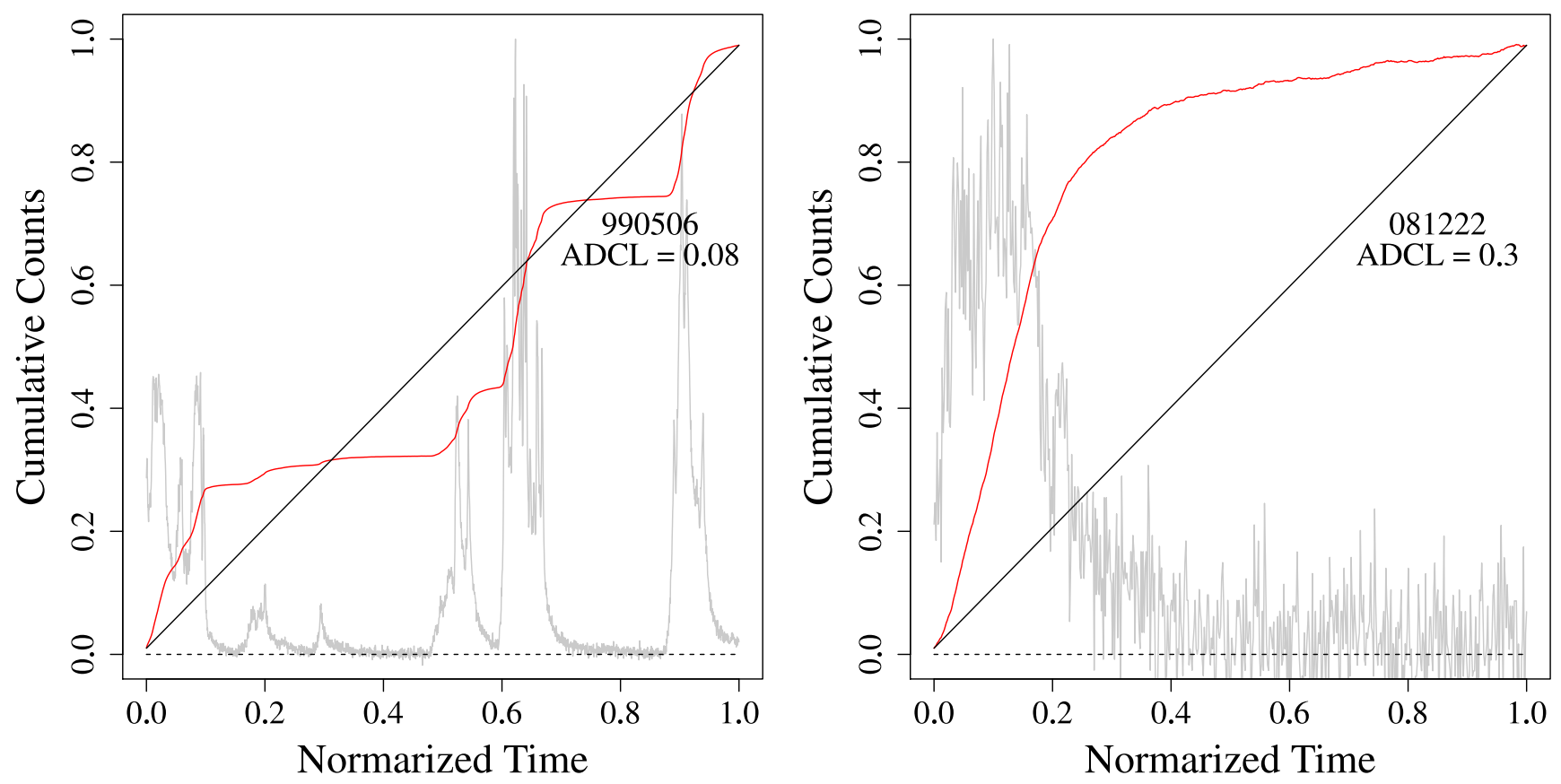

Fig. 5. left: An example of a cumulative light curve of small- $A D C L$ GRB. right : An example of a cumulative light curve of long tailed GRB.
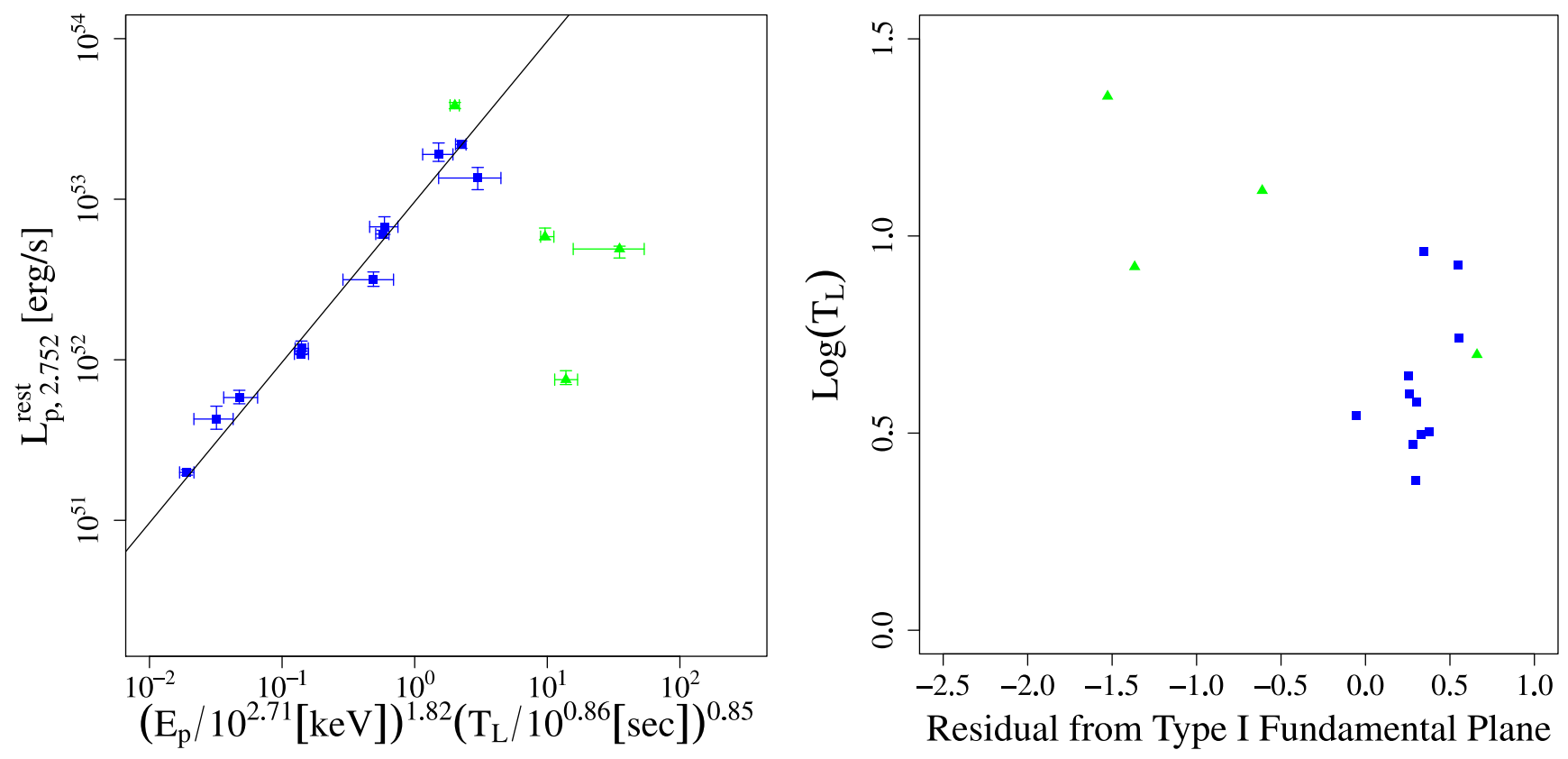

Fig. 7. left: The Fundamental Plane of large- $A D C L$ events. The blue squares represent the events with $T_{\mathrm{L}}<12$ sec used to estimate the Type II Fundamental Plane and the green triangles outliers eliminated from chi square analysis. The solid line represents the equation (12). right: The relationship between residual from the equation (12) and $T_{\mathrm{L}}$ of the long tailed events. Each symbols represents same as left figure. 
Table 6. Spectropic redshifts, spectral parameters determined by the Band model, observed fluxes and fluences with energy band of detectors, and references of 44 LGRBs

\begin{tabular}{|c|c|c|c|c|c|c|c|c|}
\hline GRB & $z^{a}$ & $\alpha$ & $\begin{array}{l}E_{\mathrm{p}}^{o b s} \\
{[\mathrm{keV}]}\end{array}$ & $\beta$ & $\begin{array}{c}P_{\mathrm{p}}^{\mathrm{obs}}\left(E_{1}-E_{2}\right)^{b} \\
{\left[(\mathrm{erg} \text { or photon }) / \mathrm{cm}^{-2} \mathrm{~s}^{-1}\right]}\end{array}$ & $\begin{array}{c}S_{\text {obs }}\left(E_{3}-E_{4}\right)^{c} \\
{\left[\mathrm{erg} / \mathrm{cm}^{-2}\right]}\end{array}$ & \multicolumn{2}{|c|}{ detector $^{d}$ reference $^{e}$} \\
\hline$\overline{970228}$ & 0.695 & $-1.54_{-0.08}^{+0.08}$ & $115.00_{-38.00}^{+38.00}$ & $-2.50_{-0.40}^{+0.40}$ & $\left(3.70_{-0.10}^{+0.10}\right) \times 10^{-6}(40-700)$ & $\left(1.10_{-0.10}^{+0.10}\right) \times 10^{-5}(40-700)$ & $\mathrm{SAX} / \mathrm{K}$ & 1 \\
\hline 971214 & 3.418 & $-0.36_{-}^{+}$ & $182.60_{-14.30}^{+11.00}$ & $-2.10_{-0.90}^{+0.52}$ & $1.95_{-0.05}^{+0.05}(50-300)$ & $\left(4.96_{-0.07}^{+0.07}\right) \times 10^{-6}(100-300)$ & BAT & 2 \\
\hline 980425 & 0.0085 & $-0.97_{-8}^{+\ell}$ & $54.90_{-11.50}^{+11.50}$ & $-2.06_{-0.09}^{+0.09}$ & $0.96_{-0.05}^{+0.05}(50-300)$ & $\left(1.67_{-0.07}^{+0.07}\right) \times 10^{-6}(100-300)$ & BAT & 2 \\
\hline 990123 & 1.6 & $-0.18_{-0.07}^{+0.08}$ & $513.00_{-21.90}^{+19.20}$ & $-2.33_{-0.09}^{+0.08}$ & $16.41_{-0.12}^{+0.12}(50-300)$ & $\left(8.72_{-0.02}^{+0.02}\right) \times 10^{-5}(100-300)$ & BAT & 2 \\
\hline 990506 & 1.3 & $-0.9_{-0.13}^{+0.19}$ & $320.70_{-38.20}^{+30.10}$ & $-2.08_{-0.10}^{+0.08}$ & $18.56_{-0.13}^{+0.13}(50-300)$ & $\left(5.16_{-0.02}^{+0.02}\right) \times 10^{-5}(100-300)$ & BAT & 2 \\
\hline 990510 & 1.619 & $-0.71_{-0.12}^{+0.12}$ & $205.50_{-12.30}^{+9.60}$ & $-2.79_{-6.21}^{+0.51}$ & $8.17_{-0.08}^{+0.08}(50-300)$ & $\left(8.04_{-0.08}^{+0.08}\right) \times 10^{-6}(100-300)$ & BAT & 2 \\
\hline 990705 & 0.843 & $-1.05_{-0.21}^{+0.21}$ & $189.00_{-15.00}^{+15.00}$ & $-2.20_{-0.10}^{+0.10}$ & $\left(3.70_{-0.10}^{+0.10}\right) \times 10^{-6}(40-700)$ & $\left(7.50_{-0.80}^{+0.80}\right) \times 10^{-5}(40-700)$ & $\mathrm{SAX} / \mathrm{K}$ & 1 \\
\hline 990712 & 0.434 & $-1.88_{-0.07}^{+0.07}$ & $65.00_{-11.00}^{+11.00}$ & $-2.48_{-0.56}^{+0.56}$ & $\left(1.30_{-0.10}^{+0.10}\right) \times 10^{-6}(40-700)$ & $\left(6.50_{-0.30}^{+0.30}\right) \times 10^{-6}(40-700)$ & $\mathrm{SAX} / \mathrm{K}$ & 1 \\
\hline 991216 & 1.02 & $-0.66_{-0.04}^{+0.04}$ & $536.50_{-20.40}^{+1.50}$ & $-2.44_{-0}^{+0}$ & $67.52_{-0.23}^{+0.23}(50-300)$ & $\left(6.37_{-0.01}^{+0.01}\right) \times 10^{-5}(100-300)$ & BAT & 2 \\
\hline 021211 & 1.01 & $-0.86^{+}$ & $45.56_{-6.23}^{+7.84}$ & $-2.18_{-0}^{+0}$ & $30.00_{-1.32}^{+1.32}(2-400)$ & $\left(3.53_{-0.21}^{+0.21}\right) \times 10^{-6}(2-400)$ & $\mathrm{HE} / \mathrm{K}$ & 3 \\
\hline 030329 & 0.168 & $-1.26^{-}$ & $67.86_{-2}^{+2}$ & $-2.28_{-0}^{+0}$ & $72.21_{-2.86}^{+2.86}(30-400)$ & $\left(1.08_{-0.01}^{+0.01}\right) \times 10^{-4}(30-400)$ & $\mathrm{HE} / \mathrm{K}$ & 3 \\
\hline 050401 & 2.9 & $-0.90_{-0.30}^{+0.30}$ & $117.50_{-18}^{+18}$ & $-2.55_{-0}^{+0}$ & $10.70_{-0.92}^{+0.92}(15-150)$ & $\left(8.22_{-0.16}^{+0.16}\right) \times 10^{-6}(15-150)$ & SK & 4 \\
\hline 050525 & 0.606 & -1.01 & $81.20_{-2.30}^{+2.30}$ & $-3.26_{-6}^{+6}$ & $41.70_{-0.94}^{+0.94}(15-150)$ & $\left(1.53_{-0.02}^{+0.02}\right) \times 10^{-5}(15-150)$ & SK & 4 \\
\hline 050603 & 2.821 & -1.03 & $343.7_{-}^{+}$ & $-2.03_{-}^{+}$ & $21.50_{-1.07}^{+1.07}(15-150)$ & $\left(6.36_{-0.23}^{+0.23}\right) \times 10^{-6}(15-150)$ & SK & 4 \\
\hline 061007 & 1.261 & -0.7 & 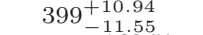 & $-2.61_{-}^{+}$ & $\left(1.66_{-0.12}^{+0.16}\right) \times 10^{-5}(20-10000$ & $\left(2.49_{-0.07}^{+0.10}\right) \times 10^{-4}(20-10000)$ & $\mathrm{K}$ & 5 \\
\hline 070125 & 1.547 & $-1.1_{-0.05}^{+0.06}$ & $367.00_{-3}^{+3}$ & $-2.08_{-}^{+}$ & $\left(1.41_{-0.14}^{+0.14}\right) \times 10^{-5}(20-10000)$ & $\left(1.74_{-0.09}^{+0.11}\right) \times 10^{-4}(20-10000)$ & $\mathrm{K}$ & 6 \\
\hline 071003 & 1.6044 & $-1.22_{-0.04}^{+0.04}$ & $1,307.00_{-381.00}^{+381.00}$ & $-9.27_{-0.73}^{+7.14}$ & $6.30_{-0.24}^{+0.24}(15-150)$ & $\left(8.30_{-0.18}^{+0.18}\right) \times 10^{-6}(15-150)$ & $\mathrm{SW} / \mathrm{K}$ & 7 \\
\hline 071010B & 0.947 & $-1.34_{-0.47}^{+0.47}$ & $45.00_{-7}^{+4}$ & $-2.34_{-c}^{+c}$ & $7.70_{-0.06}^{+0.06}(15-150)$ & $\left(4.40_{-0.06}^{+0.06}\right) \times 10^{-6}(15-150)$ & $\mathrm{SW} / \mathrm{S}$ & 7 \\
\hline 080319B & 0.937 & $-0.822_{-0.01}^{+0.01}$ & $651.00_{-8.51}^{+7.90}$ & $-3.87_{-0.66}^{+0.27}$ & $\left(1.67_{-0.10}^{+0.10}\right) \times 10^{-5}(20-7000)$ & $\left(5.72_{-0.08}^{+0.09}\right) \times 10^{-4}(20-7000)$ & $\mathrm{K}$ & 8 \\
\hline 080413B & 1.1 & $-1.24_{-0.26}^{+0.26}$ & $67.00_{-8.00}^{+13.00}$ & $-2.77_{-0.27}^{+0.22}$ & $18.70_{-0.49}^{+0.49}(15-150)$ & $\left(3.20_{-0.06}^{+0.06}\right) \times 10^{-6}(15-150)$ & $\mathrm{SW} / \mathrm{S}$ & 7 \\
\hline 080721 & 2.602 & $-0.933_{-0.05}^{+0.06}$ & $485.00_{-35.87}^{+40.73}$ & $-2.43_{-0.26}^{+0.15}$ & $\left(1.15_{-0.11}^{+0.11}\right) \times 10^{-5}(20-5000)$ & $\left(8.38_{-0.36}^{+0.38}\right) \times 10^{-5}(20-5000)$ & $\mathrm{K}$ & 9 \\
\hline 080916A & 0.689 & $\begin{array}{l}-0.11 \\
-1.11_{-0.11}^{+0.11}\end{array}$ & $129.30_{-23.30}^{+35.87}$ & $-2.49_{-0.53}^{+0.53}$ & $4.50_{-0.70}^{+0.70}(25-1000)$ & $\left(1.50_{-0.50}^{+0.50}\right) \times 10^{-5}(25-1000)$ & GBM & 10 \\
\hline 081121 & 2.512 & $-0.77^{-}$ & $248.00_{-19.45}^{+23.10}$ & $-2.51_{-0.40}^{+0.19}$ & $\left(1.94_{-0.03}^{+0.03}\right) \times 10^{-6}(20-7000)$ & $\left(1.79_{-0.19}^{+0.22}\right) \times 10^{-5}(20-7000)$ & $\mathrm{K}$ & 11 \\
\hline 081222 & 2.77 & $-0.91_{-}^{+}$ & $150.50_{-1}^{+1}$ & $-2.28_{-0}^{+0}$ & $7.70_{-0.12}^{+0.12}(15-150)$ & $\left(4.80_{-0.06}^{+0.06}\right) \times 10^{-6}(15-150)$ & GBM & 10 \\
\hline 090323 & 3.57 & $-0.96_{-c}^{+c}$ & $416.00_{-4}^{+4}$ & $-2.09_{-0}^{+0}$ & $\left(5.17_{-0.56}^{+0.57}\right) \times 10^{-6}(20-10000$ & $\left(2.02_{-0.15}^{+0.17}\right) \times 10^{-4}(20-10000)$ & $\mathrm{K}$ & 12 \\
\hline 090328 & 0.736 & -1.12 & $738.10_{-6}^{+6}$ & $-2.77_{-0}^{+0}$ & $18.50_{-0.50}^{+0.50}(8-1000)$ & $\left(8.09_{0.06}^{+0.06}\right) \times 10^{-5}(8-1000)$ & GBM & 10 \\
\hline 090424 & 0.544 & -0.9 & $149.00_{-}^{+}$ & -2.62 & $118.45_{-4.32}^{+4.32}(8-1000)$ & $\left(5.20_{-0.10}^{+0.10}\right) \times 10^{-5}(8-1000)$ & GBM & 10 \\
\hline 090618 & 0.54 & -0.87 & $149.10^{-}$ & -2.37 & $73.40_{-2.00}^{+2.00}(8-1000)$ & $\left(2.70_{-0.06}^{+0.06}\right) \times 10^{-4}(8-1000)$ & GBM & 10 \\
\hline 090902B & 1.822 & -0.85 & $792.10_{-1}^{+1}$ & $-3.80^{-}$ & $46.10_{-0.30}^{+0.30}(50-10000)$ & $\left(3.74_{-0.03}^{+0.03}\right) \times 10^{-4}(50-10000)$ & GBM & 10 \\
\hline 090926B & 1.24 & -0.11 & 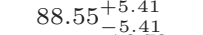 & -2.98 & $3.20_{-0.18}^{+0.18}(15-150)$ & $\left(7.30_{-0.12}^{+0.12}\right) \times 10^{-6}(15-150)$ & GBM & 10 \\
\hline 090926A & 2.1062 & -0.95 & $288.30_{-}^{+}$ & $-2.40_{-}^{+}$ & $80.80_{-0.40}^{+0.40}(8-1000)$ & $\left(1.45_{-0.04}^{+0.04}\right) \times 10^{-4}(8-1000)$ & GBM & 10 \\
\hline 091003 & 0.8969 & $-1.04_{-}^{+}$ & $398.40_{-36.90}^{+36.90}$ & $-3.58_{-1}^{+1}$ & $31.80_{-0.40}^{+0.40}(8-1000)$ & $44) \times 10^{-5}(8-1000)$ & GBM & 10 \\
\hline 091127 & 0.49 & -1.41 & 36.30 & -2.20 & ${ }_{0}^{0}(8-1000)$ & $\left.\begin{array}{l}02 \\
02\end{array}\right) \times 10^{-5}(8-1000)$ & GBM & 10 \\
\hline 091208B & 1.063 & -1 & $124.70_{-}^{\overline{+}}$ & -2.15 & $(8-1000)$ & $20) \times 10^{-6}(8-1000)$ & GBM & 10 \\
\hline $100414 \mathrm{~A}$ & 1.368 & -0 & 572. & -3.7 & $(8-1000)$ & $02) \times 10^{-4}(8-1000)$ & GBM & 10 \\
\hline 100728B & 2.106 & $-0.93_{-0.24}^{+0.24}$ & 1 & -2.23 & $20(8-1000)$ & $\left.\begin{array}{l}10 \\
10\end{array}\right) \times 10^{-6}(8-1000)$ & GBM & 10 \\
\hline $100814 \mathrm{~A}$ & 1.44 & $-0.74_{-0.13}^{+0.24}$ & $130.40_{-}^{+-}$ & $-2.73_{-0}^{+0}$ & $4.50_{-0.20}^{+0.20}(10-1000)$ & $\left(1.98_{0.06}^{+0.06}\right) \times 10^{-5}(10-1000)$ & GBM & 10 \\
\hline $100906 \mathrm{~A}$ & 1.727 & -1.4 & $113.80_{-}^{+}$ & -2.01 & $14.45_{-0.29}^{+0.29}(10-1000)$ & $.03) \times 10^{-5}(10-1000)$ & GBM & 10 \\
\hline 101219B & 0.55 & -0.63 & $63.32_{-1}^{+1}$ & $-2.46_{-}^{+r}$ & $2.00_{-0.20}^{+0.20}(10-1000)$ & $\left(5.50_{-0.40}^{+0.40}\right) \times 10^{-6}(10-1000)$ & GBM & 10 \\
\hline $110213 \mathrm{~A}$ & 1.46 & -1.24 & $49.64_{-\varepsilon}^{+\varepsilon}$ & $-2.08_{-}^{+\gamma}$ & $17.70_{-0.50}^{+0.50}(10-1000)$ & $\left(1.03_{-0.03}^{+0.03}\right) \times 10^{-5}(10-1000)$ & GBM & 10 \\
\hline $110422 \mathrm{~A}$ & 1.77 & $-0.65_{-}^{+}$ & $152.00_{-}^{+}$ & $-2.96_{-}^{+\gamma}$ & $\left(1.01_{-0.08}^{+0.08}\right) \times 10^{-5}(20-2000)$ & $\left(8.56_{-0.01}^{+0.01}\right) \times 10^{-5}(20-2000)$ & K & 13 \\
\hline $110503 \mathrm{~A}$ & 1.613 & $-0.98_{-}^{\overline{+}}$ & $219.00_{-}^{+}$ & $-2.75_{-}^{+\gamma}$ & $\left(8.59_{-0.52}^{+0.52}\right) \times 10^{-6}(20-5000)$ & $\left.\frac{2}{2}\right) \times 10^{-5}(20-5000)$ & K & 14 \\
\hline $110715 \mathrm{~A}$ & 0.82 & $-1.23_{-0}^{+0}$ & $120.00_{-6}^{+7}$ & $-2.70_{-0.30}^{+0.12}$ & $\left(7.06_{0.39}^{+0.39}\right) \times 10^{-6}(20-10000)$ & $\left(2.30_{-0.12}^{+0.12}\right) \times 10^{-5}(20-10000)$ & $\mathrm{K}$ & 15 \\
\hline $110731 \mathrm{~A}$ & 2.83 & $-0.67_{-0.15}^{+0.15}$ & $282.10_{-34.80}^{+34.80}$ & $-2.64_{-0.42}^{+0.42}$ & $20.90_{-0.50}^{+0.50}(10-1000)$ & $\left(2.22_{-0.01}^{+0.01}\right) \times 10^{-5}(10-1000)$ & GBM & 10 \\
\hline
\end{tabular}

a Taken from the J. Greiner's GRB table and references therein (http://www.mpe.mpg.de/ jcg/grbgen.html).

$b$ Observed peak photon (or energy) fluxes between $E_{1}$ to $E_{2} \mathrm{keV}$.

c Observed fluences between $E_{3}$ to $E_{4} \mathrm{keV}$.

$d$ Detectors that provide spectral parameters and light curves (SAX=BeppoSAX, K=Konus, BAT=BATSE, HE $=$ HETE-II, S=Swift, SK $=$ Swift and Konus, SW= Swift and WAM)

e References for spectral parameters, peak fluxes and fluences : (1) Amati et al. (2002) ; (2) Yonetoku et al. (2004) ; (3) Sakamoto et al. (2005) ; (4) Krimm et al. (2006) ; (5) Golenetskii et al. (2006) ; (6) Golenetskii et al. (2007) ; (7) Krimm et al. (2009) ; (8) Golenetskii et al. (2008a) ; (9) Golenetskii et al. (2008b) ; (10) This work ; (11) Golenetskii et al. (2008c) ; (12) Golenetskii et al. (2009) ; (13) Golenetskii et al. (2011a) ; (14) Golenetskii et al. (2011b) ; (15) Golenetskii et al. (2011c) 
Table 7. Intrinsic properties of 36 LGRBs

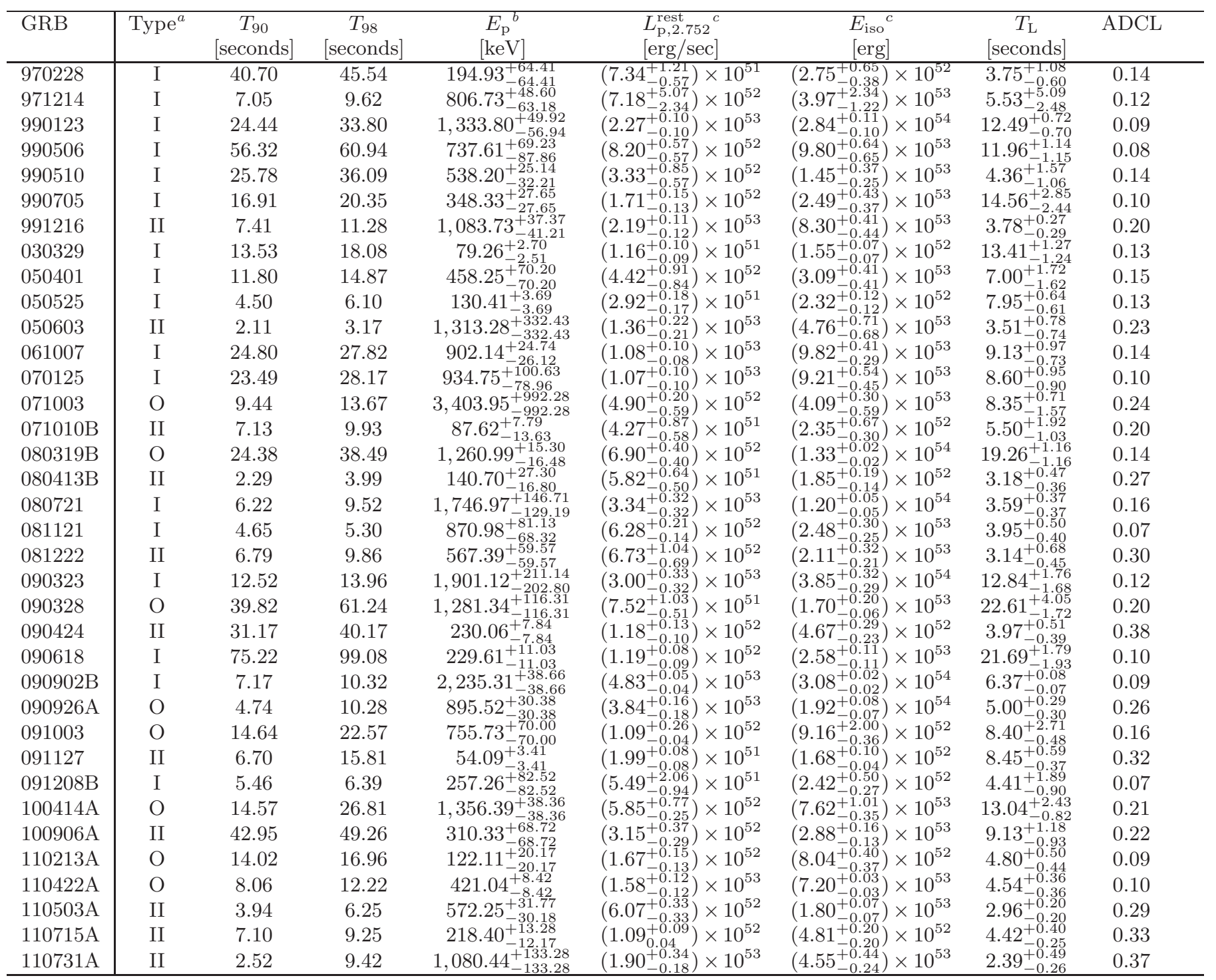

a I : Small- $A D C L$ GRBs consistent with the Type I Fundamental Plane.

II : Long tailed GRBs consistent with the Type II Fundamental Plane.

O : Outliers.

b $\quad E_{\mathrm{p}}=(1+z) E_{\mathrm{p}}^{\mathrm{obs}}$

c Integrated between $1-10,000 \mathrm{keV}$ in GRB rest frame. The flat $\Lambda \mathrm{CDM}$ universe with $H_{0}=70 \mathrm{~km} \mathrm{~s}^{-1} \mathrm{Mpc}^{-1}$ and $\Omega_{\mathrm{M}}=0.30$ is assumed. 